\title{
Integrating addiction research
}

\author{
A recent proposal to integrate addiction research portfolios from across the National Institutes of Health into a single \\ institute makes scientific sense, but the implementation will require care.
}

T he past decade has seen substantial advances in the neurobiology of addiction. We now know that multiple drugs of abuse exert common effects on the brain circuits that mediate reward, such as the dopaminergic projections from the ventral tegmental area to the nucleus accumbens. Modifications of these reward circuits, such as altered plasticity in the nucleus accumbens, may be critical for mediating addiction to a number of substances, including the compulsive consumption of natural rewards such as food. Recent work also points to potential common genetic factors that contribute to addiction. Given the wealth of data suggesting that there are likely common pathways that mediate addiction, whether to drugs or to natural rewards, the recent recommendation that the US National Institutes of Health (NIH) establish a new institute focused on substance use, abuse and addiction-related research and dissolve the National Institute on Alcohol Abuse and Alcoholism (NIAAA) and the National Institute on Drug Abuse (NIDA) is a welcome attempt to restructure one of the largest sources of addiction funding to best reflect the current state of scientific knowledge. However, the implementation of such large-scale reorganization will be tricky, so care is required to ensure that the final plan optimizes the potential benefits for addiction research.

Although the restructuring of these two major institutes may seem radical, the NIH has recognized for some time that addiction research calls for a more holistic approach. NIDA currently funds research into a wide range of drugs of abuse, including tobacco and prescription medications. At present, only alcohol research is segregated into its own institute, although there are also a few other addiction-related research programs funded by other institutes, such as the National Cancer Institute and the National Institute of Neurological Disorders and Stroke. It makes good scientific sense to bring all of these portfolios together in a single administrative entity so that research can be more easily integrated across the range of abused substances. It has even been suggested that the study of addictions be further broadened and that research into overeating and gambling (much of which is currently funded by the National Institutes of Mental Health) might also be incorporated into the new institute. The integration of addiction studies could help researchers to focus on the universal characteristics associated with the disorder.

With increased focus on a common problem, perspectives from a wide variety of researchers could have significant benefits. A diversity of viewpoints could lead to new ideas about addiction research and increased collaboration could also encourage some concrete improvements by combining distinct approaches to similar questions. For example, the NIAAA has had a great deal of success in organizing its patient database, in some measure because of the relative ease of dealing with alcoholics and their relative abundance. These resources may help to improve NIDA clinical trials. At the same time, NIDA has a reputation for aggressively screening a wide range of candidate treatments and some of these high-throughput approaches could help yield more potential therapies for alcoholism. Although some of these benefits could be reaped by a trans-NIH initiative, structural integration of these complementary programs would better help researchers capitalize on the strengths of both institutes.

This administrative consolidation, nonetheless, does raise some potentially thorny financial issues. Although the NIAAA budget is currently only half the size of the NIDA budget, it is critical that in creating a new institute, funding successful research from both institutes remains a priority. Initial plans may maintain funding levels by simply moving programs with their associated budgets to the new institute and this would be a good short-term solution. However, the long-term goal must be to structure the new institute in a way that energizes crosssubstance research synergies, but still achieves monetary efficiencies. Another economic concern is where the money for this reorganization will come from. It would be a shame to see money taken away from research to fund administrative changes without improving the longterm funding outlook for research. Even if the restructuring ultimately results in cost savings, there is a significant chance that money conserved by administrative consolidation could wind up lost to budget cuts and not fed back into research. On the other hand, these savings might be necessary just to preserve current funding levels at a time when NIH budgets are coming under increasing pressure to downsize.

The proposed integration of substance use, abuse and addiction research promises substantial benefits, but the form of the new institute remains somewhat uncertain. As befits such a significant reorganization, the NIH is currently in the process of an extended evaluation to determine the fate of the many different programs that will be affected by the restructuring. It is expected that program personnel will accompany their portfolios to their new homes, so there is a reasonable expectation of stability. Still, as of now, the new institute has no name or mission statement and the process of finding a director will surely be lengthy. These decisions will be critical determinants of the success of the new institute, so great care must be taken to ensure that the fundamental aspects of the new organization are well grounded. Although we will have to wait and see the detailed outline of the restructuring, this is a unique opportunity to reshape an institution to reflect cutting-edge science. One can only hope that putting science in the forefront will encourage the funding of the best possible addiction research and that good science will not fall victim to bad policy decisions. 